tion ( $17 / 26$ failed; mean assay $82 \%, 78 \%$ to $87 \%)$ was compounded by serious instability, with a mean loss of $17 \%$ (13\% to $22 \%)$ after 4.8 months in paired samples. The figure shows the results of the paired analysis. Full data are available on request. ${ }^{3}$

\section{Comment}

Serious instability occurred only with ergometrine injection. This result is in line with two earlier longitudinal studies during transport to and within tropical countries, in which only ergometrine injection, methylergometrine injection, and retinol capsules showed a loss of active ingredient; 12 other essential drugs were stable. ${ }^{45}$ As all three studies focused on essential drugs suspected of being unstable we conclude that, even under the most adverse tropical conditions, clinically relevant instability of essential drugs is rare. Poor initial quality (as with ampicillin, retinol, and ergometrine in our study) poses a much more serious problem as it could, in principle, occur with any drug.

The practical implication of this conclusion is that careful selection of suppliers and quality control at the entry point of the distribution chain are essential to ensure drug quality. Even in tropical climates subsequent quality checks at the district level are not necessary.

Contributors: $\mathrm{HH}$ had the original idea for the study, supervised the design and data analysis, and edited the paper; he will act as guarantor of the study. HN designed the study, collected the samples and the data, performed the data analysis, and wrote the first draft of the paper. Ms Tapuwa Muchemwa coordinated and supervised the laboratory analyses.

Funding: Swedish International Development Authority/ Swedish Agency for Research Cooperation with Developing Countries (SIDA/SAREC) and the WHO Action Programme on Essential Drugs.

Conflict of interest: None.

1 Walker GJA, Hogerzeil HV, Hillgren U. Potency of ergometrine in tropical countries. Lancet 1988;ii:393.

Hogerzeil HV, Walker GJA. Instability of (methyl)ergometrine in tropical climates-an overview. Eur J Obs Gyn Reprod Biol 1996;69:25-9.

3 Nazerali H, Hogerzeil HV. Stability of essential drugs in tropical climates. Inland stability study Zimbabwe. Geneva: World Health Organisation, 1996. (WHO/DAP/94.16.)

4 Hogerzeil HV, De Goeje MJ, Abu Reid IO. Stability of essential drugs in Sudan. Lancet 1991;338:754-5.

5 Hogerzeil HV, Battersby A, Srdanovic V, Stjernstrom NE. Stability of essential drugs during shipment to the tropics. BMJ 1992;304:210-2.

(Accepted 5 May 1998)

\title{
Blood pressure and ageing: longitudinal cohort study
}

\author{
John M Starr, Susan Inch, Susan Cross, William J MacLennan, Ian J Deary
}

Arterial blood pressure rises throughout most of life in industrialised societies. ${ }^{1}$ In old age, however, blood pressure-especially diastolic-falls. ${ }^{1}$ This may be because people who are included in epidemiological studies have diseases or take drugs that affect blood pressure. As greater disease free life expectancy is predicted, the prevalence of hypertension in the elderly may rise if a continued blood pressure increase occurs in those who remain healthy. The few studies examining this provide no consensus. ${ }^{23}$ We investigated whether blood pressure changes in old age relate to health.

\section{Subjects, methods, and results}

We visited 602 (237 men, 365 women) untreated, healthy subjects (mean age 75.7 years, range $70-88$ years) at home. ${ }^{4}$ Educational attainment was recorded and occupation classed as standard occupational classification groups 1-4 (white collar occupations) and groups 5-9 (blue collar occupations). ${ }^{5}$ Blood pressure was measured after the subjects had rested for 25-30 minutes. (A longer version of the methods appears on our web site.)

At follow up (median period 4.20 years, range 3.23-5.23 years), 69 subjects (11.5\%) had died and 105 $(17.4 \%)$ were unavailable. ${ }^{4}$ Health status (documented and self reported) was recorded for the remaining 426 subjects $(70.8 \%)$ and blood pressure remeasured at home. Measurements were of questionable reliability in 41 subjects $(9.6 \%)$ for reasons such as interruptions. Of the remaining 385 subjects (90.4\%), men had significantly lower systolic $(\mathrm{P}=0.01)$ and diastolic $(\mathrm{P}=0.017)$ pressures than women. Occupation significantly influenced diastolic $(\mathrm{P}=0.009)$, but not systolic
$(\mathrm{P}=0.68)$, pressure. Education correlated neither with systolic $(\mathrm{P}=0.44)$ nor diastolic $(\mathrm{P}=0.44)$ pressure. Age correlated negatively with diastolic $(r=-0.124$, $\mathrm{P}=0.01)$, but not with systolic $(r=-0.039, \mathrm{P}=0.2)$, pressure.

At follow up, 195 subjects (50.6\%; 69 men, 126 women) remained healthy and untreated. Correlations between baseline and follow up for systolic and diastolic pressure were 0.49 and 0.24 respectively. When baseline blood pressure was controlled for, neither age nor education significantly affected blood pressure at follow up. When systolic and diastolic pressures were combined in a multivariate repeated measures analysis of variance, sex had no significant effect $(P=0.43)$, but a significant effect was shown for occupation $(\mathrm{P}=0.021)$. A consistent pattern of rises in blood pressure occurred across occupational groups (table) except in groups 8 and 9 . When subjects were stratified by occupation-white collar $(\mathrm{n}=119)$ and blue collar $(\mathrm{n}=66)$-a significant rise in blood pressure occurred in white collar $(\mathrm{P}<0.001)$, but not blue collar $(\mathrm{P}=0.08)$, workers.

Incident disease (excluding 29 subjects with hypertension only) was reported or recorded in 163 subjects (42.3\%; 76 men, 87 women). These subjects were older $(\mathrm{P}=0.005)$ than those who remained healthy and more commonly men $(\mathrm{P}=0.021)$, but there were no significant differences in education $(\mathrm{P}=0.40)$ or occupation $(\mathrm{P}=0.067)$. Mean blood pressure remained almost unchanged (table). No significant effects were detected for sex $(\mathrm{P}=0.46)$ or occupation $(\mathrm{P}=0.37)$ over time. Compared with healthy subjects there was a significant change in systolic pressure $(\mathrm{P}=0.003)$ and diastolic pressure $(\mathrm{P}=0.016)$ over time, corrected for age.
Geriatric Medicine Unit, University of Edinburgh EH3 9EW John M Starr, senior lecturer Susan Inch, research nurse Susan Cross, research nurse William J MacLennan, professor

Department of Psychology, University of Edinburgh EH8 9JZ Ian J Deary, professor

Correspondence to: Dr John M Starr, Royal Victoria Hospital, Edinburgh EH4 2DN

John.Starr@ed.ac.uk

BMJ 1998;317:513-4

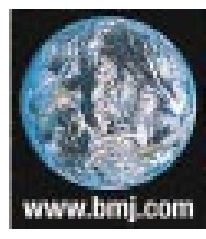

Additional data can be found on our web site 
Systolic and diastolic arterial blood pressure by standard occupational group at baseline and four years' follow up of healthy, untreated elderly people (mean age at baseline 74.4 years, mean education 11.2 years) compared with those with incident disease (mean age at baseline 75.8 years, mean education 10.9 years) or hypertension only (mean age at baseline 75.6 years, mean education 10.6 years). Values are mean (SD) unless stated otherwise

\begin{tabular}{|c|c|c|c|c|c|}
\hline \multirow[b]{2}{*}{ Group } & \multirow{2}{*}{$\begin{array}{c}\text { No of } \\
\text { patients }\end{array}$} & \multicolumn{2}{|c|}{ Baseline blood pressure $(\mathrm{mm} \mathrm{Hg})$} & \multicolumn{2}{|c|}{ Follow up blood pressure $(\mathrm{mm} \mathrm{Hg}$} \\
\hline & & Systolic & Diastolic & Systolic & Diastolic \\
\hline Unclassified & 8 & $171(21)$ & $92(4)$ & $164(19)$ & $92(10)$ \\
\hline 1 & 34 & $155(19)$ & $83(10)$ & $162(21)$ & $89(10)$ \\
\hline 2 & 31 & $152(26)$ & $82(11)$ & $157(24)$ & $83(9)$ \\
\hline 3 & 14 & $139(13)$ & $82(9)$ & $150(17)$ & $86(11)$ \\
\hline 4 & 40 & $148(20)$ & $84(10)$ & $160(22)$ & $87(8)$ \\
\hline 5 & 15 & $157(12)$ & $85(6)$ & $163(17)$ & $88(10)$ \\
\hline 6 & 8 & $156(14)$ & $83(7)$ & $159(12)$ & $91(6)$ \\
\hline 7 & 26 & $159(24)$ & $82(8)$ & $164(20)$ & $86(11)$ \\
\hline 8 & 6 & $138(23)$ & $83(11)$ & $137(10)$ & $80(4)$ \\
\hline 9 & 11 & $157(24)$ & $88(11)$ & $156(18)$ & $88(11)$ \\
\hline Healthy & 195 & $153(22)$ & $84(9)$ & $159(20)$ & $87(10)$ \\
\hline Diseased & 163 & $160(23)$ & $85(10)$ & $159(22)$ & $86(11)$ \\
\hline Hypertension only & 29 & $181(18)$ & $96(12)$ & $169(18)$ & $90(10)$ \\
\hline
\end{tabular}

\section{Comment}

In the elderly, changes in blood pressure relate to health. In those who remained healthy blood pressure continued to rise by $1.5 \mathrm{~mm} \mathrm{Hg}$ per year but fell in those who developed disease (representing probable heterogeneous effects of different medical conditions). In the healthy group no rise was seen in retired blue collar workers. Possibly, retired manual workers have more unrecognised disease, consistent with a persistent effect of socioeconomic factors on health status in old age. For healthy individuals the most important predictor of blood pressure at follow up was baseline blood pressure, accounting for $24 \%$ of the variance of systolic, but only $6 \%$ of the variance of diastolic, pressure. In general, disease affected systolic more than diastolic pressure. Hence the poorer correlation between baseline and follow up diastolic pressure compared with systolic pressure is not easily explained by possible undetected disease in the healthy group and deserves further investigation.

We thank the patients and general practitioners.

Contributors: JMS designed and supervised the study, collected and analysed the data, interpreted the results, and reported the findings; he will act as guarantor for the paper. WJMacL and IJD designed and supervised the study, interpreted the results, and reported the findings. SI and SC collected the data, interpreted the results, and reported the findings.

Funding: This study was supported by a grant from the chief scientist's office, Scotland.

Conflict of interest: None

1 Starr JM, Bulpitt CJ. Hypertension. In: Ebrahim S, ed. Epidemiology of old age. London: BMJ publications, 1996.

2 Landahl S, Bengtsson C, Sigurdsson JA, Svanborg A, Svardsudd K. Agerelated changes in blood pressure. Hypertension 1986;8:1044-9.

3 Bush TL, Linkens R, Maggi S, Hale WE. Blood pressure changes with ageing: evidence for a cohort effect. Ageing 1989;1:39-45.

Starr JM, Deary IJ, Inch S, Cross S, MacLennan WJ. Age-associated cognitive decline in healthy old people. Age Ageing 1997;26:295-300.

5 Office of Population Censuses and Surveys. Standard occupational classifcation. London: HMSO, 1991.

(Accepted 26 March 1998)

\section{Short stature and Helicobacter pylori infection in Italian children: prospective multicentre hospital based case-control study}

Clinica Pediatrica, Università di Torino, 28100 Novara, Italy Giuseppina Oderda, researcher

Elena Chiorboli, fellow in paediatrics G Bona, associate professor

Unità Operativo Epidemiologia, Centro per lo Studio e la Prevenzione Oncologica,

Azienda

Ospedaliera

Careggi, 50131

Florence, Italy

D Palli,

medical epidemiologist C Saieva,

fellow in public health

Correspondence to: Dr Oderda oderda@med. no.unipmn.it

Members of the study group are listed at the end of the article

BMJ 1998;317:514-5

\author{
Giuseppina Oderda, D Palli, C Saieva, Elena Chiorboli, G Bona on behalf of the Italian Study Group \\ on Short Stature and $H$ pylori
}

Helicobacter pylori is mainly acquired in childhood, ${ }^{1}$ but the diseases associated with such infection remain unknown. Scottish and Italian schoolchildren infected with $H$ pylori showed reduced growth in height, ${ }^{23}$ and $H$ pylori gastritis was found in $55 \%$ of French children examined for short stature. ${ }^{4}$ To evaluate the role of $H$ pylori and socioeconomic factors on growth we compared children with idiopathic short stature with those of normal height.

\section{Subjects, methods, and results}

This study was approved by and conducted within the guidelines of the gastric disease section of the Italian Society for Paediatric Gastroenterology and Hepatology (SIGEP). Between April 1996 and March 1997 we recruited 134 consecutive children aged 5-13 years (median 9.8 years) whose height was below the third centile-that is, two standard deviations below the mean height of their peers-from 26 paediatric gastroenterology and endocrinology units in Italy. We individually matched them with children of the same age and sex from the same region whose height was above the 25th centile and who had been referred for minor diseases. Obvious medical reasons for short stature (chronic or neoplastic disease with or without genetic abnormalities) were excluded by history and appropriate tests. Information on risk factors was collected by structured questionnaires with questions on socioeconomic status (education, number of cohabiting relatives, and number of rooms at home) and anthropometric data. Weight and height were measured and serum samples collected.

Serum was tested for $H$ pylori IgG in a central laboratory by enzyme linked immunosorbent assay (ELISA) (Helori, Eurospital, Italy). The assay was concurrently validated in 127 children of similar age whose $H$ pylori status was known from the results of gastric biopsy. Children were considered to be infected when their titre was $>12 \mathrm{AU} / \mathrm{ml}$, the cut off point determined from receiver operating characteristic curves (sensitivity $87 \%$, specificity $94 \%$ ). The Wilcoxon rank sum test was used to evaluate differences in continuous variables. Categorical data analysis was used on matched sets. Conditional logistic regression models were used for multivariate matched analyses. 